\title{
Dicom Viewer on Mobile and Smart Phones
}

\author{
M Yazdi ${ }^{1, *} ;$ S Mardanisamani ${ }^{2}$; M Zolfaghari ${ }^{3}$ \\ ${ }^{1}$ Professor, Signal and Image Processing Lab, Faculty of Electrical and Computer Engineering, Shiraz University, Shiraz, Iran \\ ${ }^{2}$ Signal and Image Processing Lab, Department of Electrical and Computer Engineering, Shiraz University, Shiraz, Iran \\ ${ }^{3}$ PhD Candidate, Department of Electrical and Computer Engineering, Yazd University, Yazd, Iran \\ * Corresponding author: M Yazdi, Professor, Signal and Image Processing Lab, Faculty of Electrical and Computer Engineering, Shiraz University, Shiraz, Iran, E-mail: yazdi.me@gmail.com
}

Received: 11 Dec 2016

Accepted: 01 Jan 2017

Epub: 23 Feb 2017

Ppub: 15 Jan 2018

\begin{abstract}
Background: Recent developments in sensing and communication technology have led to an explosion in the use of mobile devices such as smartphones and tablets. Dicom is used worldwide to store, exchange, and transmit medical images. Dicom images are essential in modern diagnostics. Rapid and easy availability of these images right at the point of care is a crucial factor.

Objectives: We created a mobile application (App) to access Dicom images anywhere and anytime. Since, such existing mobile Apps are unable to connect to our Kpacs radiology devices, we designed a specific mobile App that can effectively be connected to our Kpacs.

Methods: There is an increasing need for developing applications on mobile phones to view and treat medical images of all kinds. Dicom, as a medical image standard, is used in nearly all medical centers and hospitals. In addition, Dicom viewers have already been developed on computer networks to serve physicians and hospital staffs to view, process, and manipulate Dicom images. Therefore, having Dicom viewers on mobile and smart phones can greatly help physicians for rapid access to medical images anytime and anywhere. We have used Java, Android, and OpenCV programming to create a native Dicom viewer App. Our App is for primary processing and preservation of medical images in Dicom format. This App is equipped with most common tools for manipulation of Dicom images and has the advantage of being accessible to everyone.

Results: Dicom viewer App includes some benefit features: such as opening and saving medical images in Dicom format, brightness/contrast controlling, performing zoom and pan on Dicom images, having medical image processing operations, and being suitable for patients to show Dicom images on their own mobile phone.

Conclusion:This important technology provides physicians with the ability to immediately view images and make diagnoses without needs to be back at workstation. Physicians are now able to access images at home while they are on background duty or just in every place in the hospital. Moreover, even patients may view and interact with their personal images on their own mobile phone.

Keywords: mHealth; Dicom Images; Mobile App; Java Programming
\end{abstract}

